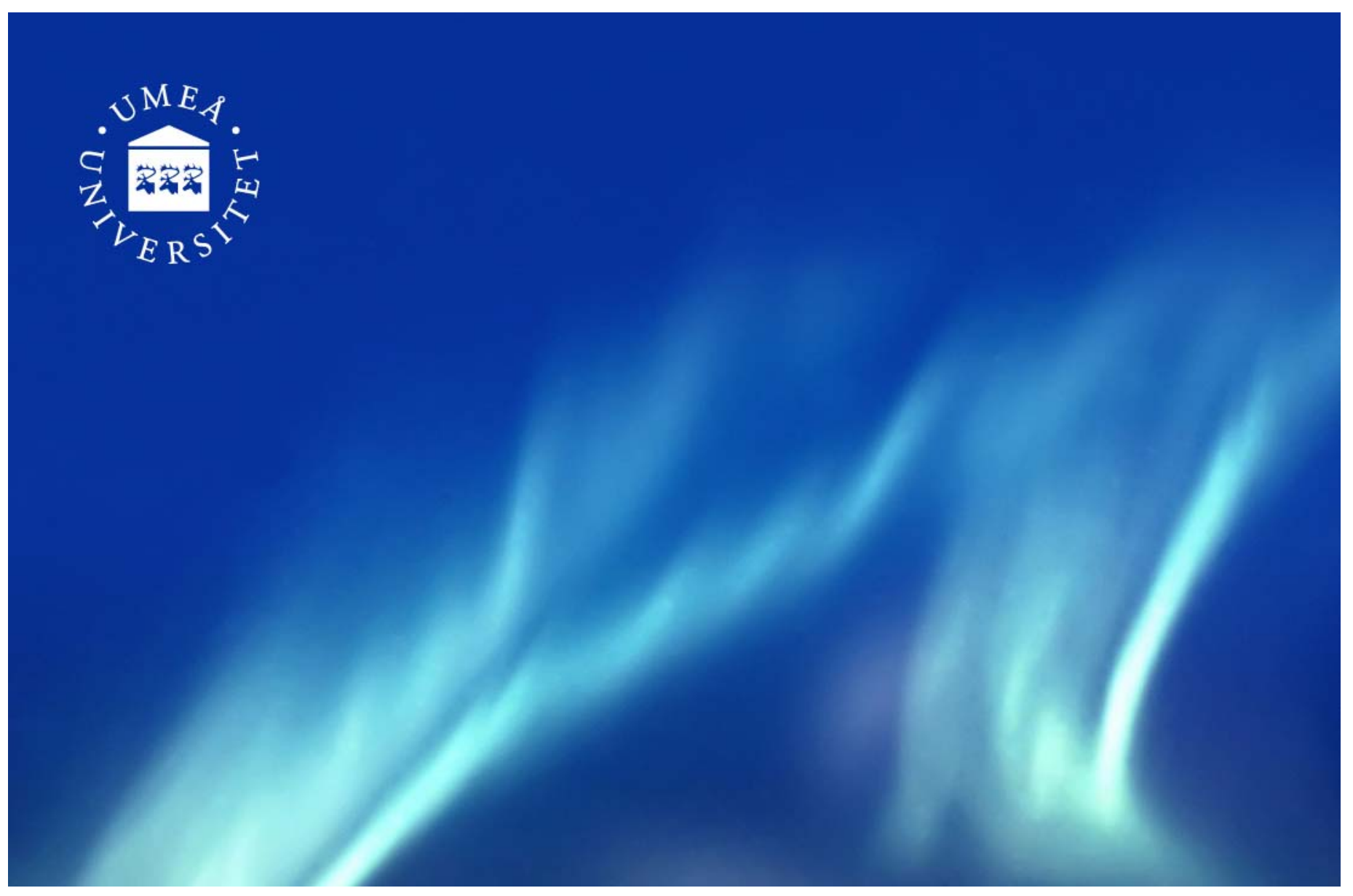

DiVA - Digitala Vetenskapliga Arkivet http://umu.diva-portal.org

This is the pre-peer reviewed version of the following article:

Sundstedt, S., Olofsson, K., van Doorn, J., Linder, J., Nordh, E. et al. (2012)

"Swallowing function in Parkinson's patients following Zona Incerta deep brain stimulation"

Acta Neurologica Scandinavica

The article has been published in final form at:

URL: http://dx.doi.org/10.1111/j.1600-0404.2012.01658.x

Access to the published version may require subscription.

Permanent link to this version:

http://urn.kb.se/resolve?urn=urn:nbn:se:umu:diva-55468 


\title{
Swallowing function in Parkinson's patients following Zona Incerta deep brain stimulation
}

Stina Sundstedt, MSc ${ }^{1}$, Katarina Olofsson, MD PhD ${ }^{2}$, Jan van Doorn, $\mathrm{PhD}^{1}$, Jan Linder, $\mathrm{MD}^{3}$, Erik Nordh, $\mathrm{MD} \mathrm{PhD}^{3,} \&$ Patric Blomstedt, $\mathrm{MD} \mathrm{PhD}^{3}$,

\footnotetext{
${ }^{1}$ Department of Clinical Sciences, Division of Speech and Language Pathology;

${ }^{2}$ Department of Clinical Sciences, Division of Otorhinolaryngology;

${ }^{3}$ Department of Pharmacology and Clinical Neuroscience, Division of Clinical Neuroscience,

Umeå University, Umeå, Sweden.
}

\author{
Correspondence to: \\ Stina Sundstedt \\ Department of Clinical Sciences, \\ Division of Speech and Language Pathology, \\ Umeå University, \\ SE-901 87 Umeå, Sweden; \\ +46739516522, +358407397393. \\ stina.sundstedt@gmail.com
}




\section{Abstract \\ Objective}

The purpose of the present study was to examine if there was a negative effect of caudal Zona Incerta deep brain stimulation (cZI DBS) on pharyngeal swallowing function in Parkinson's patients (PD). There are no former reports on swallowing and cZI DBS.

\section{Methods}

Eight patients (aged 49 to 71 years; median 62) were evaluated pre- and postoperatively, at six and 12 months after DBS surgery. Evaluation tools were Fiberoptic Endoscopic Evaluation of Swallowing examinations and patients' self-assessments of the swallowing function including a visual analogue scale and quality of life related questions. The swallowing protocol included Rosenbeck's Penetration-Aspiration Scale, Secretion Severity Scale and parameters for pre-swallow spillage, pharyngeal residue and pharyngeal clearance.

\section{Results}

There was no clear-cut effect of neurostimulation postoperatively at six and 12 months on any of the swallowing parameters except for pre-swallow spillage which was slightly worsened in the stimulation on condition $12 \mathrm{~m}$ postoperatively. The answers to the self assessment questions did not vary significantly.

\section{Conclusions}

The effect of the stimulation on swallowing function varied among individuals but the overall outcome was that cZI DBS did not seem to have a negative influence on swallowing function in the eight patients studied.

Key words: Deep brain stimulation, caudal Zona Incerta, dysphagia, Parkinson's disease, FEES, swallowing function, L-dopa, aspiration 


\section{Introduction}

Dysphagia is an important deficit in patients with idiopathic Parkinson's disease (PD) (1), which notably increases the mortality risk ${ }^{2}$, and aspiration pneumonia caused by dysphagia has been reported to have the highest mortality rate among comorbidities in PD (2). While the swallowing act can be divided into four phases (pre-oral, oral, pharyngeal and oesophageal), aspiration is mainly associated with deterioration in the pharyngeal phase. Dysphagia affects quality of life, as even mild swallowing problems have psychosocial effects for patients with PD and their carers (3). It is thus essential to take dysphagia into account when considering treatment alternatives (3).

The most common treatment alternative for PD patients is levodopa (L-Dopa) medication which improves the general motor symptoms (4). However, the effect of L-dopa on swallowing function is yet not clearly elucidated (5). Some studies report a slight decline in function (6) while others report small improvements $(5,7)$. The risk of aspiration does not seem to be affected by L-dopa $(5,6)$.

Deep brain stimulation (DBS) in the subthalamic nucleus (STN) is an established therapy for PD but also the posterior subthalamic area (PSA) including the caudal Zona Incerta (cZI), has been suggested as an alternative target in PD (8-12). Improvements in global motor function and in health-related quality of life are seen in STN DBS (13-17). Similar improvements have been seen in cZI DBS $(10,11,18)$ and a non-randomized sequential study has suggested cZI DBS to be more effective than STN DBS with regard to limb motor outcomes (11).

The effect of STN DBS on swallowing function has been evaluated in a single study, using video fluoroscopy (19). STN DBS improved some aspects of the pharyngeal stage of deglutition, while the oral stage was unaffected. Aspiration and penetration were evident both on and, to a larger extent, off stimulation, but without reaching significant levels (19). Dysphagia has further been reported in a few patients as a complication to STN DBS (13, 17, 20, 21). In a long-term follow up study, dysphagia was also reported as an adverse effect to STN DBS surgery in $15 \%$ of the patients (22). The effect of cZI DBS on swallowing function has not yet been described in the literature, apart from one case of transient dysphagia due to a misplaced electrode (18). Considering this, it is of importance not only to evaluate the effects of cZI DBS on limb motor outcomes but also regarding swallowing function. 
The purpose of the study was to compare postoperative (6 \& 12 months) swallowing function, on and off stimulation, with a preoperative baseline in order to identify possible negative effects on swallowing function.

\section{Materials and methods}

Eight consecutive patients (six males, age 49 - 71 years; median 62) undergoing bilateral cZi DBS for PD were included. . The selection for surgery was based on overall motor function and no consideration was taken to swallowing. The mean duration of disease was $6.1 \pm 2.8$ years. The median UPDRS III scores preoperatively was 35.5 (range 29-58) OFF medication and 20.0 (range 10-42) ON medication. The surgical procedure and the target have previously been described in detail (23).

All postoperative examinations were made with a clinically optimized dose of L-dopa medication. Any changes in the patients' medication or stimulator settings were initiated and motivated by the clinical situation, as judged by a neurologist specialised in movement disorders. The oropharyngeal aspects were not especially taken into consideration. A study design that included a postoperative "Stimulator $O F F$, Medication $O F F$ " state, which would have enabled separate evaluation of the effects of medication and stimulation on swallowing, was considered not ethically defensible due to the considerable discomfort.

The study was approved by the Regional Ethical Review Board in Umeå (08-0934M).

\section{Testing conditions}

Patients were evaluated pre- and postoperatively, at six and 12 months after surgery. At the preoperative assessment the patients were examined on medication (1.5 times the ordinary dose) and off medication (medications withheld for approximately 12 hours. The postoperative evaluations were performed within the optimal time in the patient's usual medication cycle, and with stimulation on and off (after $60 \mathrm{~min}$ of stimulation on and off, respectively) (Table 1). 


\section{Evaluation of swallowing}

The Fiberoptic Endoscopic Evaluation of Swallowing (FEES) examinations were conducted using an Olympus ENF P4 transnasal flexible endoscope and a Wolf endocam 5502. The patients were asked to swallow a test meal consisting of one solid and four different liquid consistencies, all coloured with green dye, with each bolus size estimated as one tablespoonful $(10 \mathrm{ml})$. The liquid consistencies were $50 \mathrm{ml}$ water $\left(\mathrm{C}_{1}\right), 50 \mathrm{ml}$ water and $5 \mathrm{ml}$ jellification powder $\left(\mathrm{C}_{2}\right), 50 \mathrm{ml}$ water and $10 \mathrm{ml}$ jellification powder $\left(\mathrm{C}_{3}\right)$ and 50 $\mathrm{ml}$ water and $15 \mathrm{ml}$ jellification powder $\left(\mathrm{C}_{4}\right)$, while the solid consistency $\left(\mathrm{C}_{5}\right)$ was a biscuit with a smear of $\mathrm{C}_{4}$ on top. The order of consistencies in examination was primarily $\mathrm{C}_{2}, \mathrm{C}_{3}$, $\mathrm{C}_{4}, \mathrm{C}_{1}$ and $\mathrm{C}_{5}$.

After the test meal, the patients were asked to evaluate their swallowing function, by putting a mark on a linear visual analogue scale, where one point represents $100 \%$ functional swallowing while the other represents total loss of swallowing function. This was used as measurement of the percentage deterioration in swallowing function.

All video recordings were de-identified and randomly ordered before the swallowing function was scored according to a predefined protocol. Fifteen percent of the recordings were further evaluated by another author for interrater reliability. The protocol included Rosenbeck's Penetration-Aspiration Scale (24) (scale range1-8 points), and a Secretion Severity Scale (25) (scale range 0-3). Pre-swallow spillage, pharyngeal residue and pharyngeal clearance were analyzed and evaluated according to Table 2 . In one of the patients, a small part of the video recording was lost and the missing scores were replaced by estimates from the research notes made during the FEES-examination.

The patients also answered a custom made questionnaire regarding Quality of Life related aspects of swallowing at three time points (before surgery, 6 and 12 months after surgery). The questionnaire has seven items: 1) affected swallowing, 2) consistency modification, 3) weight loss, 4) coughing when eating, 5) decreased mealtime pleasure, 6) sticky saliva/hemming and 7) drooling. Possible scores: zero to three points (the lower score the better function). At the commencement of the study no Swedish version of the validated SWAL-QOL 1.0 was available (26). 


\section{Data and Statistical analysis}

Prior to analyses, a mean Penetration/ Aspiration score from the five different food consistencies was calculated, in order to enable statistical comparisons concerning possible effects of testing condition. The scores from Penetration/Aspiration scale, pharyngeal residue, pharyngeal clearance and pre-swallow spillage were transformed so that the lowest score was always 0. E.g. a mean Penetration/Aspiration score of $0 \mathrm{p}$ meant that there was no penetration or aspiration for any consistency. The maximum possible transformed score for Penetration/Aspiration was $7 \mathrm{p}$. Pharyngeal residue, pre-swallow spillage and clearance each had a full range of 0-1 and Secretion Scale ranged between 0-3.

Friedman repeated measures test by ranks was used to discover statistically significant differences between conditions with Wilcoxon signed rank test for pair wise post-hoc testing. A p-value $<0.05$ was considered as statistically significant.

\section{Results}

Group medians and means for the different swallowing scores in specified testing conditions are seen in Table 3. Results from the Friedman test by ranks are also seen in the table. Brackets show significant post-hoc differences.

Preoperatively no significant differences were found between medication OFF and test dose L-dopa condition on any of the swallowing parameters ( $p>.05)$.

Preoperative and postoperative comparisons between test dose L-dopa and six months and 12 months postoperative stimulation $\mathrm{ON}$ and OFF revealed a significant reduction of preswallow spillage at six months in both ON and OFF condition $(z=-2.27, p=.02, \mathrm{z}=-2.047$, $p=.04)$.

Postoperatively at six months no differences were found between stimulation $\mathrm{ON}$ and stimulation OFF conditions on the different swallowing parameters ( $p>.05)$. At 12 months postoperatively there was significantly more pre-swallow spillage in the stimulation ON condition than in the OFF condition $(z=-2.070, p=.04)$. There was no significant differences for the other swallowing parameters $(p>.05)$.

Individual descriptive data of Penetration/Aspiration score are presented in Table 4. Regardless of testing condition, the highest Penetration/Aspiration score noted in the group 
was $5 \mathrm{p}$. The full range of the Penetration/Aspiration scale is 1-8p. No aspiration was observed in any patient.

Intrarater reliability was $95 \%$ and Kappa scores for intrarater reliability and interrater reliability were .88 and .72 .

\section{Self-assessments}

The self-reported assessments of percentage deterioration in swallowing function are presented in Table 3. There were no significant differences between testing conditions (Table 3). Similarly, there were no significant differences in the responses to the short questionnaire between the testing conditions (Table 5).

\section{Discussion}

There are no former reports on the effect of cZI DBS on swallowing function. The present study examined the effect of cZI DBS on pharyngeal swallowing function in a heterogeneous group of patients with PD. Even if the number of participants is acceptable given the clinical population and the number of operations performed, the number is low and the results must be interpreted in light of this.

The main finding at the one-year follow up was that cZI DBS did not significantly increase the prevalence of penetration or aspiration, nor was there any increased occurrence of pharyngeal residue or decreased clearance due to the stimulation or the operation itself.

This is of importance, as the data show that a cZI DBS therapy optimized for minimizing a patient's overall motor disturbances does not significantly impair these aspects of the swallowing function. This is essential in clinical practice, as patients with PD are often troubled by dysphagia (1), which both increases the mortality risk and affects quality of life $(3,27)$.

Pre-swallow spillage, as a sign of uncoordinated oral and pharyngeal phase in PD patients, has been reported as a risk factor for aspiration (28). In this report the oral phase was not evaluated. Pre-swallow spillage was the only swallowing parameter that was significantly affected by the surgery itself and by the stimulation. The surgery itself seemed to improve the pre-swallow spillage as the incidence of pre-swallow spillage was lower six months after the surgery in the stimulation OFF condition than in the test dose L-dopa condition. 
At 12 months postoperatively the stimulation seemed to have a possible negative impact on the pre-swallow spillage as there was more pre-swallow spillage in the 12 months postoperative stimulation ON condition than in the OFF condition. However, the amount of pre-swallow spillage at 12 months postoperative stimulation $\mathrm{ON}$ did not differ significantly from the preoperative test dose L-dopa condition.

The results show a relatively low prevalence of penetration, and there was no observable aspiration in the studied group of patients with mean disease duration $6.1 \pm 2.8$ years. However, in an earlier study it was shown that aspiration is evident mainly in the later stages of the disease (29), and the latency of the onset of dysphagia might be up to 130 months (10 yrs) after onset of PD (30). The patients in the present study were on the verge of this latency time, and hence may not yet have manifested such problems as aspiration.

The variability in the self-reported visual analogue scale assessment of swallowing function was large and no significant differences were found. Since neither the assessment of percentage deterioration in swallowing function nor the answers to the short questionnaire varied significantly among testing conditions it can be said that for patients in the current study cZI DBS did not alter the subjective experience of swallowing related aspects of quality of live.

In the studied group somewhat limited by low numbers and with mild to moderate preoperative swallowing divergences we conclude that cZI DBS did not have a negative outcome on the swallowing function. It is important to confirm these findings using larger materials, and to evaluate the impact of cZI DBS in later stages of the disease, where swallowing might be more severely affected, and perhaps more liable to external influence in the form of DBS surgery. 


\section{Acknowledgements}

We are all deeply indebted to Anna Fredricks and Anders Asplund for administrative and technical support. We wish to acknowledge the support of grants from Magnus Bergvall's Foundation and the Swedish Parkinson's Fund. A visiting professorship at the Rehabilitation Research Chair, King Saud University, Riyadh, Saudi Arabia, to one of the authors (E.N.) is gratefully acknowledged.

\section{Conflict of Interest and Sources of Funding}

Stina Sundstedt: none

Katarina Olofsson: none

Jan van Doorn: none

Dr Linder served on the scientific advisory board for Glaxo Smith Kline and H Lundbeck and received lecture honoraria from Medtronic Inc., Solvay, Orion Pharma, UCB Pharma and Nordic Infu Care.

Dr Erik Nordh received lecture honoraria from IPSEN and Pfizer.

Patric Blomstedt: none 


\section{References}

1 JANKOVIC J. Parkinson's disease: clinical features and diagnosis. J Neurol Neurosur Ps 2008;79:368-76.

2 FERnANDEZ HH, LAPANE KL. Predictors of mortality among nursing home residents with a diagnosis of Parkinson's disease. Med Sci Monit 2002;8:CR241-6.

3 Miller N, Noble E, Jones D, Burn D. Hard to swallow: dysphagia in Parkinson's disease. Age ageing 2006;35:614-8.

4 HORNYKIEWICZ O. A brief history of levodopa. J Neurol 2010;257:249-52.

5 Hunter P, Crameri J, Austin S, Woodward M, Hughes A. Response of parkinsonian swallowing dysfunction to dopaminergic stimulation. J Neurol Neurosur Ps 1997;63:579-83.

6 Lim A, LEOW L, HuCKABEe M, FRAMPTON C, ANDERSON T. A pilot study of respiration and swallowing integration in Parkinson's disease:"On" and "Off" levodopa. Dysphagia 2008;23:76-81.

7 FuH J, LEE R, WANG S et al. Swallowing difficulty in Parkinson's disease. Clin Neurol Neurosur 1997;99:106-12.

8 Blomstedt P, Sandvik U, Fytagoridis A, Tisch S. The Posterior Subthalamic area in the treament of movement disorders: past, present, and future. Neurosurgery 2009;64:1029-38.

9 Velasco F, Jimenez F, PEREZ M et al. Electrical stimulation of the prelemniscal radiation in the treatment of Parkinson's disease: An old target revised with new techniques. Neurosurgery 2001;49:293-306.

10 Kitagawa M, Murata J, Uesugi H et al. Two-year follow-up of chronic stimulation of the posterior subthalamic white matter for tremor-dominant Parkinson's disease. Neurosurgery 2005;56:281-7.

11 Plaha P, Ben-Shlomo Y, Patel N, Gill S. Stimulation of the caudal zona incerta is superior to stimulation of the subthalamic nucleus in improving contralateral parkinsonism. Brain 2006;129:1732-47.

12 CARrillo-Ruiz J, Velasco F, JimEnez F et al. Bilateral electrical stimulation of prelemniscal radiations in the treatment of advanced Parkinson's Disease.

Neurosurgery 2008;62:347-57.

13 OSTERGAARD K, SUNDE N, DUPONT E. Effects of bilateral stimulation of the subthalamic nucleus in patients with severe Parkinson's disease and motor fluctuations. Mov Disord 2002;17:693-700.

14 Obeso J, Guridi J, Rodriguez-Oroz M et al. Deep-brain stimulation of the subthalamic nucleus or the pars interna of the globus pallidus in Parkinson's disease. New Engl J Med 2001;345:956-63. 
15 Erola T, KARINEN P, HeIKKINEN E et al. Bilateral subthalamic nucleus stimulation improves health-related quality of life in Parkinsonian patients. Parkinsonism Relat D 2005;11:89-94.

16 Zibetti M, Torre E, CinQuePAlmi A et al. Motor and nonmotor symptom follow-up in Parkinsonian patients after deep brain stimulation of the subthalamic nucleus. Eur Neurol 2007;58:218-23.

17 Valldeoriola F, Pilleri M, Tolosa E, Molinuevo J, Rumia J, Ferrer E. Bilateral subthalamic stimulation monotherapy in advanced Parkinson's disease: Long-term follow-up of patients. Mov Disord 2002;17:125-32.

18 Plaha P, KHAN S, GILl S. Bilateral stimulation of the caudal zona incerta nucleus for tremor control. J Neurol Neurosur Ps 2008;79:504-13.

19 Ciucci MR, BARKMEIER-Kraemer JM, Sherman SJ. Subthalamic nucleus deep brain stimulation improves deglutition in Parkinson's disease. Mov Disord 2008;23:676-83.

20 Krause M, Fogel W, Mayer P, Kloss M, Tronnier V. Chronic inhibition of the subthalamic nucleus in Parkinson's disease. J Neurol Sci 2004;219:119-24.

21 TASSORELli C, Buscone S, SANDrini G et al. The role of rehabilitation in deep brain stimulation of the subthalamic nucleus for Parkinson's disease: A pilot study. Parkinsonism Relat D 2009;15:675-81.

22 LiAng G, ChOU K, BALtuch G et al. Long-term outcomes of bilateral subthalamic nucleus stimulation in patients with advanced Parkinson's disease. Stereotact Funct Neurosurg 2006;84:221-7.

23 Blomstedt P, SAndviK U, Tisch S. Deep Brain Stimulation in the Posterior Subthalamic Area in the Treatment of Essential Tremor. Mov Disord 2010;25:1350-6.

24 Rosenbek J, Robbins J, Roecker E, Coyle J, Wood J. A penetration aspiration scale. Dysphagia 1996;11:93-8.

25 Murray J, Langmore SE, Ginsberg S, Dostie A. The significance of accumulated oropharyngeal secretions and swallowing frequency in predicting aspiration.

Dysphagia 1996;11:99-103.

26 Swedish version of SWAL-QOL 1.0, Finizia, C,. Sahlgrenska akademin, Göteborgs Universitet.

27 Lo R, TAnner C, Albers K et al. Clinical Features in Early Parkinson Disease and Survival. Arch Neurol-Chicago 2009;66:1353-8.

28 Logemann J. Treatment for aspiration related to dysphagia: an overview. Dysphagia 1986;1:34-8.

29 LEOPOLD N, KAGEL M. Pharyngo-esophageal dysphagia in Parkinson's disease. Dysphagia 1997;12:11-8.

30 Muller J, Wenning G, Verny M et al. Progression of dysarthria and dysphagia in postmortem-confirmed Parkinsonian disorders. Arch Neurol 2001;58:259-64. 
Table 1 Testing conditions

\begin{tabular}{|c|c|c|}
\hline Surgical status & Condition & Treatment status \\
\hline \multirow{2}{*}{ Preoperative } & Medication off & L-dopa witheld for 12 hours or overnight \\
\hline & Test dose of L-dopa & 1,5 hours after a test dose of L-dopa 1,5 times higher than normal \\
\hline \multirow{2}{*}{$\begin{array}{c}6 \text { months } \\
\text { Postoperative }\end{array}$} & Stimulator off & Stimulator turned off for $60 \mathrm{~min}$. Normal dose of L-dopa. \\
\hline & Stimulator on & Stimulator turned on for $60 \mathrm{~min}$. Normal dose of L-dopa. \\
\hline \multirow{2}{*}{$\begin{array}{l}12 \text { months } \\
\text { Postoperative }\end{array}$} & Stimulator off & Stimulator turned off for $60 \mathrm{~min}$. Normal dose of L-dopa. \\
\hline & Stimulator on & Stimulator turned on for $60 \mathrm{~min}$. Normal dose of L-dopa. \\
\hline
\end{tabular}


Table 2 Evaluation of pre-swallow spillage, pharyngeal residue and pharyngeal clearance

\begin{tabular}{ll}
\hline Parameter & Operationalization \\
\hline Pre- swallow spillage & Green bolus is seen before white-out \\
Pharyngeal residue & Residue in the sinus piriformis or vallecula \\
Pharyngeal clearance & No residue after the first deglutition \\
\hline
\end{tabular}


Table 3 Scores from swallowing evaluation and self-reported assessment of swallowing function. Means of the five consistencies. Median, range, mean and standard deviation. Friedman test comparing differences among conditions. Brackets show significant post-hoc differences. The lower the scores the better function.

\begin{tabular}{|c|c|c|c|c|c|c|c|c|}
\hline \multirow{2}{*}{$\begin{array}{c}\text { Median } \pm \\
\text { Range } \\
(\text { Mean } \pm \text { SD })\end{array}$} & \multicolumn{2}{|c|}{ Preoperative } & \multicolumn{2}{|c|}{ 6m Pos toperative } & \multicolumn{2}{|c|}{ 12m Postoperative } & \multicolumn{2}{|c|}{ Friedman test $(n=8)$} \\
\hline & Med off & $\begin{array}{l}\text { Test dose } \\
\text { of L-dopa }\end{array}$ & Stim off & Stim on & Stim off & Stim on & $F r$ & $p$ \\
\hline P/A & $\begin{array}{c}0.2 \pm 2.4 \\
(0.6 \pm 0.9)\end{array}$ & $\begin{array}{l}0.4 \pm 1.6 \\
(0.6 \pm 0.7)\end{array}$ & $\begin{array}{c}0.2 \pm 1.4 \\
(0.4 \pm 0.5)\end{array}$ & $\begin{array}{l}0.3 \pm 1.8 \\
(0.5 \pm 0.7)\end{array}$ & $\begin{array}{c}0.3 \pm 0.4 \\
(0.2 \pm 0.2)\end{array}$ & $\begin{array}{l}0.1 \pm 2.0 \\
(0.4 \pm 0.7)\end{array}$ & 2.66 & $p=0.75$ \\
\hline Phr & $\begin{array}{c}0.6 \pm 1.0 \\
(0.6 \pm 0.3)\end{array}$ & $\begin{array}{c}0.5 \pm 1.0 \\
(0.6 \pm 0.4)\end{array}$ & $\begin{array}{c}0.6 \pm 1.0 \\
(0.5 \pm 0.4)\end{array}$ & $\begin{array}{l}0.3 \pm 1.0 \\
(0.4 \pm 0.4)\end{array}$ & $\begin{array}{c}0.7 \pm 1.0 \\
(0.6 \pm 0.4)\end{array}$ & $\begin{array}{l}0.8 \pm 1.0 \\
(0.6 \pm 0.5)\end{array}$ & 5.06 & $p=0.41$ \\
\hline PS & $\begin{array}{l}0.6 \pm 1.0 \\
(0.6 \pm 0.4)\end{array}$ & $\begin{array}{l}\text { 席 } \\
0.9 \pm 0.4 \\
(0.8 \pm 0.2)\end{array}$ & 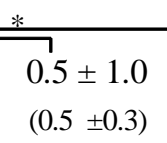 & $\begin{array}{l}7.6 \pm 0.8 \\
(0.6 \pm 0.3)\end{array}$ & $\begin{array}{c}\Gamma^{*} \\
0.7 \pm 1.0 \\
(0.6 \pm 0.3)\end{array}$ & $\begin{array}{l}7.0 \pm 1.0 \\
(0.8 \pm 0.4)\end{array}$ & 13.83 & $p=0.01$ \\
\hline $\mathrm{CC}$ & $\begin{array}{c}0.5 \pm 1.0 \\
(0.5 \pm 0.3)\end{array}$ & $\begin{array}{c}0.5 \pm 1.0 \\
(0.6 \pm 0.4)\end{array}$ & $\begin{array}{c}0.7 \pm 1.0 \\
(0.6 \pm 0.4)\end{array}$ & $\begin{array}{c}0.3 \pm 1.0 \\
(0.4 \pm 0.4) \\
*\end{array}$ & $\begin{array}{l}0.7 \pm 1.0 \\
(0.6 \pm 0.4)\end{array}$ & $\begin{array}{c}0.8 \pm 1.0 \\
(0.6 \pm 0.5)\end{array}$ & 5.25 & $p=0.39$ \\
\hline SS & $\begin{array}{l}0.5 \pm 2.0 \\
(0.8 \pm 0.9)\end{array}$ & $\begin{array}{c}1.0 \pm 2.0 \\
(0.8 \pm 0.7)\end{array}$ & $\begin{array}{c}1.5 \pm 3.0 \\
(1.38 \pm 1.3)\end{array}$ & $\begin{array}{c}0.0 \pm 2.0 \\
(0.4 \pm 0.7)\end{array}$ & $\begin{array}{l}0.5 \pm 1.0 \\
(0.5 \pm 0.5)\end{array}$ & $\begin{array}{l}0.0 \pm 1.0 \\
(0.4 \pm 0.5)\end{array}$ & 12.09 & $p=0.03$ \\
\hline$\%$ DSF & $\begin{array}{r}22.5 \pm 98.0 \\
(33.8 \pm 31.3)\end{array}$ & $\begin{array}{c}23.0 \pm 98.0 \\
(29.4 \pm 30.4)\end{array}$ & $\begin{array}{c}23.5 \pm 40.0 \\
(23.9 \pm 12.0)\end{array}$ & $\begin{array}{l}18.0 \pm 44.0 \\
(20.8 \pm 13.9)\end{array}$ & $\begin{array}{c}14.5 \pm 22.0 \\
(14.9 \pm 8.2)\end{array}$ & $\begin{array}{l}16.5 \pm 25 \\
(15.6 \pm 8.2)\end{array}$ & 4.45 & $p=0.49$ \\
\hline
\end{tabular}

P/A: Penetration and Aspiration scale, 0-8p. Phr: pharyngeal residual, 0-1p. PS: pre-swallow spillage,0-1p. CC: clearance, 0-1p. SS: Secretions scale, 0-3p. \%DSF: Self-reported percentage deterioration of swallowing function. $0-100 \% . * p<.05$, posthoc testing. 
Table 4 Individual presentation of penetration for different consistencies. Number of consistencies within two different categories of Penetration/Aspiration scale.

\begin{tabular}{|c|c|c|c|c|c|c|c|c|c|}
\hline \multicolumn{5}{|c|}{ Preoperative } & \multicolumn{5}{|c|}{ 6m Postoperative } \\
\hline & \multicolumn{2}{|c|}{ Med off } & \multicolumn{2}{|c|}{ Test dose L-dopa } & & \multicolumn{2}{|c|}{ Stim off } & \multicolumn{2}{|c|}{ Stim on } \\
\hline & Penetration & Penetration & Penetration & Penetration & & Penetration & Penetration & Penetration & Penetration \\
\hline & Mild & Vocal folds & Mild & Vocal folds & & Mild & Vocal folds & Mild & Vocal folds \\
\hline & $2-3 p$ & $4-5 p$ & $2-3 p$ & $4-5 p$ & & $2-3 p$ & $4-5 p$ & $2-3 p$ & $4-5 p$ \\
\hline Pat 1 & - & $\mathrm{C}_{2}, \mathrm{C}_{3}, \mathrm{C}_{4}$ & $\mathrm{C}_{2}, \mathrm{C}_{3}, \mathrm{c}_{4}$ & - & Pat 1 & $\mathrm{C}_{5}$ & - & $\mathrm{C}_{3}$ & - \\
\hline Pat 2 & $\mathrm{C}_{4}$ & - & $\mathrm{c} 3, \mathrm{c}_{5}$ & - & Pat 2 & $\mathrm{c}_{3}, \mathrm{c}_{4}$ & - & $\mathrm{C}_{4}$ & - \\
\hline Pat 3 & - & - & - & - & Pat 3 & $\mathrm{C}_{4}$ & - & $\mathrm{C}_{5}$ & $\mathrm{c}_{1}, \mathrm{c}_{2}$ \\
\hline Pat 4 & $\mathrm{C}_{2}-\mathrm{C}_{5}$ & - & $\mathrm{c}_{3}$ & $\mathrm{C}_{2}$ & Pat 4 & $\mathrm{C}_{2}-\mathrm{C}_{5}$ & - & $\mathrm{C}_{2}, \mathrm{C}_{3}, \mathrm{c}_{4}$ & - \\
\hline Pat 5 & - & - & - & - & Pat 5 & - & - & - & - \\
\hline Pat 6 & $\mathrm{C}_{4}$ & - & $c_{2}, c_{3}, c_{4}$ & - & Pat 6 & - & - & - & - \\
\hline Pat 7 & - & - & - & - & Pat 7 & - & - & - & - \\
\hline Pat 8 & - & - & - & - & Pat 8 & - & - & $\mathrm{C}_{4}$ & - \\
\hline
\end{tabular}

$12 \mathrm{~m}$ Postoperative

\begin{tabular}{cccccc}
\hline & \multicolumn{2}{c}{ Stimoff } & & \multicolumn{2}{c}{ Stim on } \\
\cline { 2 - 3 } \cline { 5 - 6 } & Penetration & Penetration & & Penetration & Penetration \\
& Mild & Vocal folds & & Mild & Vocal folds \\
& $2-3 p$ & $4-5 \mathrm{p}$ & & $2-3 \mathrm{p}$ & $4-5 \mathrm{p}$ \\
\hline Pat 1 & $\mathrm{c}_{2}$ & - & - & - \\
Pat 2 & $\mathrm{c}_{4}$ & - & & $\mathrm{c}_{4}$ & - \\
Pat 3 & $\mathrm{c}_{4}$ & - & & $\mathrm{c}_{2}$ & $\mathrm{c}_{3}, \mathrm{c}_{4}$ \\
Pat 4 & $\mathrm{c}_{3}$ & - & & $\mathrm{c}_{2}, \mathrm{c}_{3}$ & - \\
Pat 5 & - & - & & - & - \\
Pat 6 & $\mathrm{c}_{4}$ & - & & - & - \\
Pat 7 & - & - & & $\mathrm{c}_{3}$ & - \\
Pat 8 & - & - & & - & - \\
\hline
\end{tabular}

Penetration Mild: bolus penetrates the laryngeal vestibule above vocal folds, scale score 2-3p. Penetration Vocal folds: bolus contacts the vocal folds, scale score 4-5p. 
Table 5 Answers to a short questionnaire about swallowing function. Scores 0-3p. The lower score the better function.

\begin{tabular}{lccccc}
\hline \multicolumn{1}{c}{$\begin{array}{c}\text { Median } \pm \text { Range } \\
\text { (Mean } \pm \text { SD })\end{array}$} & Preoperative & Postoperative & \multicolumn{2}{c}{ 12m } & \multicolumn{2}{c}{ Fostoperative } & Fr & $p$ \\
\hline 1. Affected swallowing & $0.0 \pm 1.0$ & $0.5 \pm 1.0$ & $0.5 \pm 1.0$ & 0.67 & $p=0.71$ \\
& $(0.4 \pm 0.5)$ & $(0.5 \pm 0.5)$ & $(0.5 \pm 0.5)$ & & \\
2. Consistency modification & $0.0 \pm 0.0$ & $0.0 \pm 0.0$ & $0.0 \pm 1.0$ & 2.00 & $p=0.37$ \\
& $(0.0 \pm 0.0)$ & $(0.0 \pm 0.0)$ & $(0.1 \pm 0.3)$ & & \\
3. Weight loss & $0.0 \pm 1.0$ & $0.0 \pm 0.0$ & $0.0 \pm 1.0$ & 2.67 & $p=0.26$ \\
& $(0.3 \pm 0.5)$ & $(0.0 \pm 0.0)$ & $(0.3 \pm 0.5)$ & & \\
4. Coughing when eating & $0.0 \pm 2.0$ & $0.0 \pm 0.0$ & $0.0 \pm 0.0$ & 4.00 & $p=0.16$ \\
& $(0.4 \pm 0.7)$ & $(0.0 \pm 0.0)$ & $(0.0 \pm 0.0)$ & & \\
5. Decreased mealtime pleasure & $0.0 \pm 1.0$ & $0.0 \pm 2.0$ & $0.0 \pm 2.0$ & 5.00 & $p=0.78$ \\
& $(0.3 \pm 0.5)$ & $(0.3 \pm 0.7)$ & $(0.4 \pm 0.8)$ & & \\
6. Sticky saliva/Hemming & $0.5 \pm 2.0$ & $1.0 \pm 2.0$ & $0.5 \pm 1.0$ & 2.55 & $p=0.28$ \\
& $(0.6 \pm 0.7)$ & $(1.0 \pm 0.5)$ & $(0.5 \pm 0.5)$ & & \\
7. Drooling & $1.0 \pm 1.0$ & $1.0 \pm 2.0$ & $0.5 \pm 2.0$ & 0.74 & $p=0.69$ \\
& $(0.6 \pm 0.5)$ & $(0.9 \pm 0.6)$ & $(0.9 \pm 1.0)$ & & \\
\hline
\end{tabular}

